\title{
Triple-differential cross sections for $(e, 2 e)$ electron-impact ionization dynamics of tetrahydrofuran at low projectile energy
}

\author{
Enliang Wang $\odot,{ }^{1}$ Xueguang Ren $\odot,{ }^{2,1, *}$ Maomao Gong, ${ }^{3,4}$ Esam Ali $\odot,{ }^{5,6}$ Zhenpeng Wang $\odot,{ }^{3,4}$ Chao Ma, ${ }^{2,1}$ Don Madison, ${ }^{5}$ \\ Xiangjun Chen $\odot, 3,4$ and Alexander Dorn ${ }^{1}$ \\ ${ }^{1}$ Max Planck Institut für Kernphysik, Saupfercheckweg 1, 69117 Heidelberg, Germany \\ ${ }^{2}$ School of Physics, Xi'an Jiaotong University, Xi'an 710049, China \\ ${ }^{3}$ Hefei National Laboratory for Physical Sciences at Microscale and Department of Modern Physics, University of Science and \\ Technology of China, Hefei, Anhui 230026, China \\ ${ }^{4}$ Synergetic Innovation Center of Quantum Information and Quantum Physics, University of Science and Technology of China, \\ Hefei, Anhui 230026, China \\ ${ }^{5}$ Department of Physics, Missouri University of Science and Technology, Rolla, Missouri 65409, USA \\ ${ }^{6}$ Department of Natural Sciences, D. L. Hubbard Center for Innovation, Northwest Missouri State University, Maryville, Missouri 64468, USA
}

(Received 8 September 2020; accepted 1 December 2020; published 16 December 2020)

\begin{abstract}
We study the triple-differential cross section (TDCS) for the electron-impact ionization of the highest occupied molecular orbital of tetrahydrofuran at a projectile energy $E_{0}=91 \mathrm{eV}$. The experimental data were measured using a reaction microscope, which covers a large part of the full solid angle for the secondary electron emission with energies ranging from 6 to $15 \mathrm{eV}$, and projectile scattering angles ranging from $-10^{\circ}$ to $-20^{\circ}$. The experimental TDCSs are internormalized across all measured scattering angles and ejected energies. They are compared with predictions from the multicenter distorted-wave (MCDW) approximation and a modified MCDW-Nee method which includes the postcollision interaction (PCI) using the Ward-Macek factor. Additional calculations were obtained using a molecular three-body distorted-wave (M3DW) approach which accounts for PCI in an exact treatment. Generally, the MCDW-Nee and M3DW models show better agreement with experiment than the MCDW calculations. This shows the importance of accounting for PCI for low-energy outgoing electrons in electron-impact ionization processes.
\end{abstract}

DOI: 10.1103/PhysRevA.102.062813

\section{INTRODUCTION}

Electron scattering on atoms and molecules is important in many areas of science and technology ranging from astrochemistry over atmospheric sciences to plasma physics [1-3]. Also, in medical radiation therapy damage to biological tissue is caused not only by the primary radiation or particle but to a large part by the abundant and low-energetic secondary electrons with energies typically below $100 \mathrm{eV}$. It is well established that these electrons play an important role in producing DNA lesions either indirectly, e.g., by producing reactive hydroxyl radicals from the radiolysis of water, or directly via excitation and ionization reactions and by electron attachment-all of which can initiate molecular bond breaking and subsequent dissociation [4-10].

Methods to calculate and assess this radiation-induced damage on the molecular level require accurate and possibly

\footnotetext{
*renxueguang@xjtu.edu.cn
}

Published by the American Physical Society under the terms of the Creative Commons Attribution 4.0 International license. Further distribution of this work must maintain attribution to the author(s) and the published article's title, journal citation, and DOI. Open access publication funded by the Max Planck Society. highly differential interaction data. Electron-impact ionization can be characterized in a kinematically complete way in so-called $(e, 2 e)$ studies [11,12] where the two outgoing electrons are detected in coincidence and the momentum vectors of all final-state particles are obtained. The resulting triple-differential cross section (TDCS) serves as a powerful tool to test theoretical models that account for the quantum few-body dynamics and to provide the input parameters for the Monte Carlo simulations of electron track structures (see, e.g., Refs. [13-18]).

Here, we consider tetrahydrofuran (THF), which is an organic compound with the formula $\mathrm{C}_{4} \mathrm{H}_{8} \mathrm{O}$. Owing to its furanose ring structure, THF is often regarded as a molecular analog of the deoxyribose sugar ring in the DNA backbone for the modeling of ionization and fragmentation of biomolecules [19-24]. Therefore, there exist a number of experimental and theoretical studies on elastic scattering of low-energy electrons [19,25-29] as well as $(e, 2 e)$ studies [13-18]. Colyer et al. [14] and Jones et al. [15] used an impact energy of $250 \mathrm{eV}$ and an ejected-electron energy of 10 and $20 \mathrm{eV}$, respectively. The experimental data were compared with predictions of the molecular three-body distorted-wave (M3DW) model [30,31] in which reasonable agreement was obtained for the relative intensity of the binary and recoil lobes except for the smaller scattering angle $\left(\theta_{1}=5^{\circ}\right)$ measured in these experiments $[14,15]$. 
Recent calculations from a multicenter distorted-wave (MCDW) model provided an improved description of the experimental data for THF at $250 \mathrm{eV}$ concerning both binary and recoil lobes [18].

For lower-energy collisions which are of greater biological interest, the M3DW was compared with experimental data for $26.5 \mathrm{eV}$ ionization of THF with the ejected electron having an energy of $3.5 \mathrm{eV}$ and a range of scattering angle $\left(15^{\circ}, 25^{\circ}\right.$, and $35^{\circ}$ ) [17] and fairly good agreement between experiment and theory was found. Interestingly, for this energy, the spectrum did not exhibit the normal binary and recoil peaks which shows that, for low energies, the binary and recoil peaks are mixed up due to the multicenter scattering potential. To the best of our knowledge, all of the available $(e, 2 e)$ TDCS for THF were determined under coplanar kinematics where the ejected electron is detected in the scattering plane defined by the momentum vectors of the incident and the scattered projectile. Other kinematics beyond the coplanar condition are required to give a comprehensive understanding of the ionization dynamics of THF.

In this paper, we performed kinematically complete experiments for low-energy electron-impact ionization $\left(E_{0}=\right.$ $91 \mathrm{eV}$ ) of THF using a reaction microscope (REMI) technique. This allows us to gain more insight into the ionization dynamics due to the fact that TDCSs covering a large part for the full solid angle for the ejected electron can be determined [32-40]. The ionization of the highest occupied molecular orbital (HOMO) of THF was determined by coincidence detection of two outgoing electrons and the intact parent ion, i.e., $\mathrm{THF}^{+}$,

$$
e_{0}+\mathrm{THF} \rightarrow \mathrm{THF}^{+}\left(\mathrm{HOMO}^{-1}\right)+e_{1}+e_{2} .
$$

Here, $e_{i}(i=0,1,2)$ refers to the incident, the scattered projectile, and the ejected electron, respectively. As previously identified, e.g., in Ref. [22], only ionization of the HOMO is leading to the intact $\mathrm{THF}^{+}$while ionization of other orbitals gives rise to fragmentation of the residual ion. The measured TDCSs are internormalized for different kinematics, and thus a single scaling factor is sufficient to put the experimental data on an absolute scale. The kinematical conditions cover projectile scattering angles $\theta_{1}$ of $-10^{\circ},-15^{\circ}$, and $-20^{\circ}$, and ejected-electron energies $E_{2}$ of 6 and $15 \mathrm{eV}$. The ionization energy (IE) of the THF HOMO amounts to $9.8 \mathrm{eV}$ [22], and thus for $E_{0}=91 \mathrm{eV}$ the sum energy of the outgoing electrons is $E_{1}+E_{2}=81.2 \mathrm{eV}$. The experimental data are compared with theoretical predictions from both the multicenter distorted-wave model (MCDW) [41-43] and molecular three-body distorted-wave models (M3DW) [30,31].

This paper is organized as follows: After a brief description of the experiment in Sec. II, a brief introduction of the theoretical models is given in Sec. III. The comparison between experiment and theory is discussed in Sec. IV before we draw conclusions in Sec. V. Unless specified otherwise, atomic units (a.u.) are used throughout this paper.

\section{EXPERIMENTAL METHOD}

The experiments were performed using a dedicated REMI which was particularly designed for electron-impact experiments. Details about the experimental setup have been given elsewhere $[22,44,45]$. Here, a brief description is introduced. A well-focused ( 1-mm-diam) pulsed electron beam is crossed with a supersonic gas jet and nonscattered electrons were collected by the beam dump which is a central bore in the electron detector. The pulsed projectile beam is emitted from an electron gun consisting of a tantalum photocathode which is irradiated by pulsed UV light $(\lambda=266 \mathrm{~nm})$ of $0.5 \mathrm{~ns}$ duration and electrostatic focusing lens elements. The energy width of the electron pulse is about $0.5 \mathrm{eV}$ [22]. Using uniform electric and magnetic fields $(B=7.0 \mathrm{G})$ which are parallel to the projectile beam axis, the final charged state fragments, i.e., electrons as well as ions, are projected in opposite directions on two position- and time-sensitive multihit delay line detectors. The field strength for electron extraction is $E=1.5 \mathrm{~V} \mathrm{~cm}^{-1}$. After $400 \mathrm{~ns}$ when the electrons have arrived at the detector, the electric field is ramped up to $33 \mathrm{~V} \mathrm{~cm}^{-1}$ for extracting the fragment ions. The acceptance angle for detection of the electrons up to the energy of $15 \mathrm{eV}$ is almost $4 \pi$, where small forward and backward angles and small energies below $2 \mathrm{eV}$ are excluded due to the presence of the beam dump in the center of the electron detector. The collection efficiency for the intact $\mathrm{THF}^{+}$ion is almost $100 \%$.

Experimental data are recorded by triple-coincidence measurement of two outgoing electrons and the $\mathrm{THF}^{+}$ion, i.e., $e_{1}, e_{2}$, and $\mathrm{THF}^{+}$in Eq. (1). The momentum vectors of the detected electrons and ions are reconstructed from the measured time of flight and position of the particles hitting the detectors.

\section{THEORETICAL MODELS}

For comparison, TDCS calculations are performed using the MCDW and the M3DW models. The details of these two models have been described previously in Refs. [41-43] for MCDW and Refs. [30,31,46] for M3DW, respectively. Only a brief outline will be presented here.

\section{A. MCDW model}

The MCDW method is developed within the framework of the first Born approximation (FBA) in which the continuum wave function of the slow ejected electron is calculated in the spatially fixed multicenter potential of the residual ion under the sudden approximation and plane waves are used to describe the incoming projectile and scattered electron. In the usual scattering theory, the transition amplitude for a given molecular orientation in the laboratory can be expressed as

$$
T(\Omega)=\left\langle\boldsymbol{k}_{1} \Psi_{f}^{(-)}\left(\boldsymbol{k}_{2} ; \mathcal{R}_{\Omega}^{-1}(\boldsymbol{r})\right)|V(\boldsymbol{r})| \boldsymbol{k}_{0} \Psi_{i}\left(\boldsymbol{R}_{\Omega}^{-1}(\boldsymbol{r})\right)\right\rangle,
$$

where $\boldsymbol{k}_{0}, \boldsymbol{k}_{1}$, and $\boldsymbol{k}_{2}$ represent the momentum vectors of the incident, scattered, and ejected electrons, respectively. $\boldsymbol{k}_{0}$ and $\boldsymbol{k}_{1}$ describe the plane waves of the incident and scattered electrons. The molecular orientation is defined by the Euler angle $\Omega=(\alpha, \beta, \gamma) .\left|\Psi_{i}\right\rangle$ and $\left|\Psi_{f}^{(-)}\right\rangle$represent the initial bound wave function and the final state in which the ionized orbital is substituted by the continuum wave function of the ejected electron. $\boldsymbol{r}$ refers to the set of electronic coordinates. $V$ is the interaction potential of incident electron and the molecular target. With the help of the Bethe integral, Eq. (2) can be simplified as a one-electron problem under the frozen-core 
approximation,

$$
\begin{aligned}
T(\Omega) \sim & \left\langle\mathcal{F}^{(-)}\left(\boldsymbol{k}_{2} ; \mathcal{R}_{\Omega}^{-1}(\boldsymbol{r})\right)\right| e^{i \boldsymbol{K} \cdot \boldsymbol{r}} \\
& -\frac{1}{N} \sum_{n} Z_{n} e^{i \boldsymbol{K} \cdot \boldsymbol{R}_{n}}\left|\phi\left(\mathcal{R}_{\Omega}^{-1}(\boldsymbol{r})\right)\right\rangle,
\end{aligned}
$$

where $\mathcal{F}^{(-)}$and $\phi\left(\mathcal{R}_{\Omega}^{-1}(\boldsymbol{r})\right)$ are the continuum wave function of the ejected electron which is solved under the multicenter field of the recoil ion and the bound wave functions of the active electron. The term $e^{i \boldsymbol{K} \cdot \boldsymbol{r}}$ represents the scattering from the active electron in the target and the term $\sum_{n} Z_{n} e^{i \boldsymbol{K} \cdot \boldsymbol{R}_{n}} / N$ represents the scattering from the nuclei with $N$ being the number of electrons in the target. In experiment, the molecular orientation cannot be determined and an averaging over all the molecular orientations is needed theoretically to produce the TDCS,

$$
\sigma^{\mathrm{TDCS}}=\frac{1}{(2 \pi)^{5}} \frac{k_{1} k_{2}}{k_{0}} \frac{1}{8 \pi^{2}} \int|T(\Omega)|^{2} d \Omega .
$$

With low-energy electron impact, the correlation of the two outgoing electrons and residual ion will be important. The full final-state Coulomb interaction, which is normally called the postcollision interaction (PCI), can be included through the famous Gamow factor $\left(N_{e e}\right)$ [47],

$$
N_{e e}=\left|e^{-\frac{\pi \gamma}{2}} \Gamma(1-i \gamma)\right|^{2}=\frac{\pi / k_{a b}}{e^{\pi / k_{a b}}-1},
$$

where $\Gamma(1-i \gamma)$ is the gamma function, $k_{a b}=\mu v_{a b}, \mu=1 / 2$ is the reduced mass for the two electrons, $v_{a b}$ is the relative velocity between the two outgoing electrons, and $\gamma=1 / v_{a b}$ is the Sommerfeld parameter. Hereafter, the MCDW calculation without the PCI effect is referred to as MCDW while MCDWNee denotes such a calculation including the PCI effect.

\section{B. M3DW model}

For the M3DW calculation, all of the three continuum electrons are described by a distorted wave. The direct-scattering amplitude is given by

$$
T_{\mathrm{dir}}=\left\langle\chi_{1}^{-}\left(\boldsymbol{k}_{1}, \boldsymbol{r}_{0}\right) \chi_{2}^{-}\left(\boldsymbol{k}_{2}, \boldsymbol{r}_{1}\right) \boldsymbol{C}_{12}\left(\boldsymbol{r}_{01}\right)|W| \phi\left(\boldsymbol{r}_{1}\right) \chi_{0}^{+}\left(\boldsymbol{k}_{0}, \boldsymbol{r}_{0}\right)\right\rangle,
$$

where $\boldsymbol{k}_{0}, \boldsymbol{k}_{1}$, and $\boldsymbol{k}_{2}$ are the wave vectors for the initial, scattered, and ejected electrons, respectively. $\chi_{0}^{+}\left(\boldsymbol{k}_{0}, \boldsymbol{r}_{0}\right)$ is an initial-state continuum distorted wave and the $(+)$ indicates outgoing-wave boundary conditions. $\chi_{1}^{-}\left(\boldsymbol{k}_{1}, \boldsymbol{r}_{0}\right) \chi_{2}^{-}\left(\boldsymbol{k}_{2}, \boldsymbol{r}_{1}\right)$ are the scattered and ejected-electron distorted waves with incoming-wave boundary conditions, and the factor $\boldsymbol{C}_{12}\left(\boldsymbol{r}_{01}\right)$ is the final-state Coulomb-distortion factor between the two electrons normally called the PCI. The perturbation $W=$ $V_{i}-U_{i}$, where $V_{i}$ is the initial-state interaction potential between the incident electron and the neutral molecule, and $U_{i}$ represents the spherically symmetric interaction between the projectile and the active electron which is used to calculate the initial-state distorted wave $\chi_{0}^{+}\left(\boldsymbol{k}_{0}, \boldsymbol{r}_{0}\right)$. Here, $\phi\left(\boldsymbol{r}_{1}\right)$ is the initial bound-state Dyson orbital averaged over all molecular

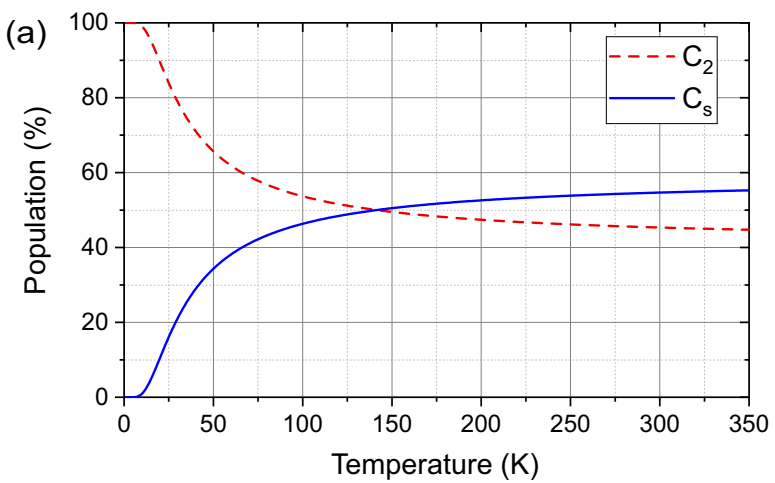

(b)

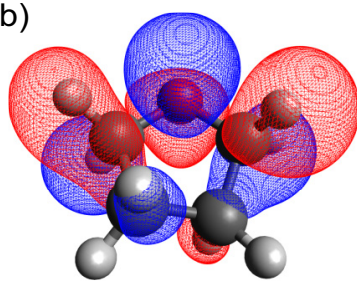

$\mathrm{C}_{2}: 9 \mathrm{~b}$

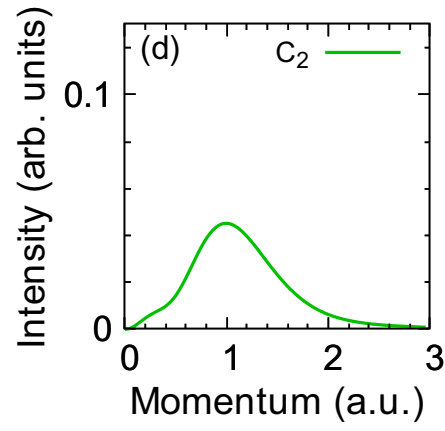

(c)

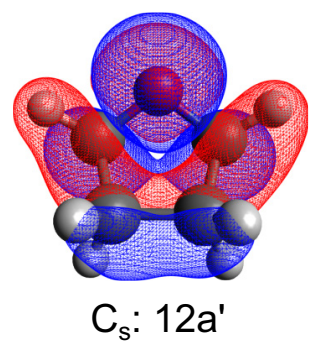

FIG. 1. (a) Relative population of two conformers $C_{2}$ and $C_{s}$ for THF as a function of temperature. Orbital images of the HOMOs for conformers (b) $C_{2}$ and (c) $C_{s}$. The electron momentum profiles for conformers (d) $C_{2}$ and (e) $C_{s}$. The equilibrium geometries were optimized by MP2/aug-cc-pVTZ and the wave functions were calculated by the Becke three-parameter Lee-YangParr (B3LYP)/aug-cc-pVTZ functional.

orientations. The TDCS can be obtained from

$$
\sigma^{\mathrm{TDCS}}=\frac{1}{(2 \pi)^{5}} \frac{k_{1} k_{2}}{k_{0}}\left[\left|T_{\mathrm{dir}}\right|^{2}+\left|T_{\mathrm{exc}}\right|^{2}+\left|T_{\mathrm{dir}}-T_{\mathrm{exc}}\right|^{2}\right],
$$

where the exchange scattering $T_{\text {exc }}$ is calculated similarly to $T_{\text {dir }}$ except that the particles 1 and 2 are interchanged in the final-state wave function.

\section{RESULTS AND DISCUSSION}

\section{A. Conformation analysis of THF}

There are two conformers for THF with $C_{2}$ and $C_{s}$ point group symmetry $[48,49]$ with slightly different bonding angles as shown in Figs. 1(b) and 1(c). In order to obtain the energies we optimized these two conformers with second-order Møller-Plesset perturbation theory (MP2) using the augmented correlation-consistent polarized valence triple zeta (aug-cc-pVTZ) basis set. The population 
distributions can be determined from the energy difference of these two conformers and the temperature with the Boltzmann distribution [48],

$$
f=g e^{\frac{-E_{t}}{k_{B} T}}
$$

where $g$ is the symmetry factor which is 1 for each of the conformers, and $k_{B}$ and $T$ are the Boltzmann constant and the temperature, respectively. $E_{t}$ is the total energy including the electronic energy $\left(E_{e}\right)$, zero-point energy (ZPE), as well as the Gibbs free energy $(G)$, i.e., $E_{t}=E_{e}+\mathrm{ZPE}+G$. The Gibbs free energy is defined by $G=H-T S$, where $H$ and $S$ are enthalpy and entropy, respectively. The zeropoint energy, enthalpy, and entropy are determined by the frequency calculation which is performed by the same method as the geometry optimization. The population distribution as a function of temperature of the two conformers is shown in Fig. 1(a). Approaching $0 \mathrm{~K}$, the $C_{2}$ conformer is the stablest one whose population decreases with increasing temperature. The branching ratio of the $C_{2}$ to $C_{s}$ conformer is about $45 \%$ : $55 \%$ at room temperature, which is consistent with the previous studies [48]. In this work, the averaged temperature of the target is estimated to be $30 \mathrm{~K}$ by analyzing the recoil ion momenta of $\mathrm{THF}^{+}$. The branching ratio of $80 \%: 20 \%$ for the $C_{2}$ to $C_{s}$ conformer is obtained. Figures 1(b) and 1(c) show the optimized geometries of the $C_{2}$ and $C_{s}$ conformers for THF and orbital images of their HOMOs calculated using the Gaussian package [50]. It can be seen from Figs. 1(b) and 1(c) that the HOMOs are composed of oxygen $2 p$ lone-pair orbitals. The calculated electron momentum profiles are presented in Figs. 1(d) and 1(e), which show $p$ - and $s$ - $p$-type momentum profiles for the HOMO of the $C_{2}$ and $C_{s}$ conformers, respectively.

\section{B. General comparison of M3DW and MCDW approaches}

The calculated TDCSs for the HOMO of each conformer are presented in Fig. 2 for the scattering plane under two kinematics. One is close to the Bethe-ridge condition with $\theta_{1}=-20^{\circ}(q=0.91$ a.u. $)$ and $E_{2}=15 \mathrm{eV}\left(\mathrm{k}_{2}=1.05\right.$ a.u. $)$ [see in Figs. 2(b) and 2(d)]. For the Bethe-ridge condition the magnitude of the ejected-electron momentum is roughly equal to the momentum transfer $q$ and, thereby, the reaction is most likely due to a collision of the two free electrons while the residual ion acts as a spectator [51]. Under this kinematic condition, the binary peak shows generally a minimum for the $p$-type orbital (i.e., the HOMO of $C_{2}$ ) or a maximum for the $s$-p-type orbital (i.e., the HOMO of $C_{s}$ ) along the momentum transfer $q$ direction [11], while for the kinematics falling below the Bethe-ridge condition, i.e., $\theta_{1}=-10^{\circ}$ ( $q=0.56$ a.u.) and $E_{2}=15 \mathrm{eV}\left(k_{2}=1.05\right.$ a.u.) in Figs. 2(a) and 2(c), the binary peak shows generally a maximum for both $p$ - and $s$-p-type orbitals [11]. This behavior is seen in the results of the MCDW calculations. For M3DW, however, the calculated TDCSs for the HOMO of the $C_{2}$ and $C_{s}$ conformers are almost identical to each other. This illustrates the fact that the orientation-averaged molecular orbital method used in the M3DW calculations may smear out the characteristics of the ionized orbitals.
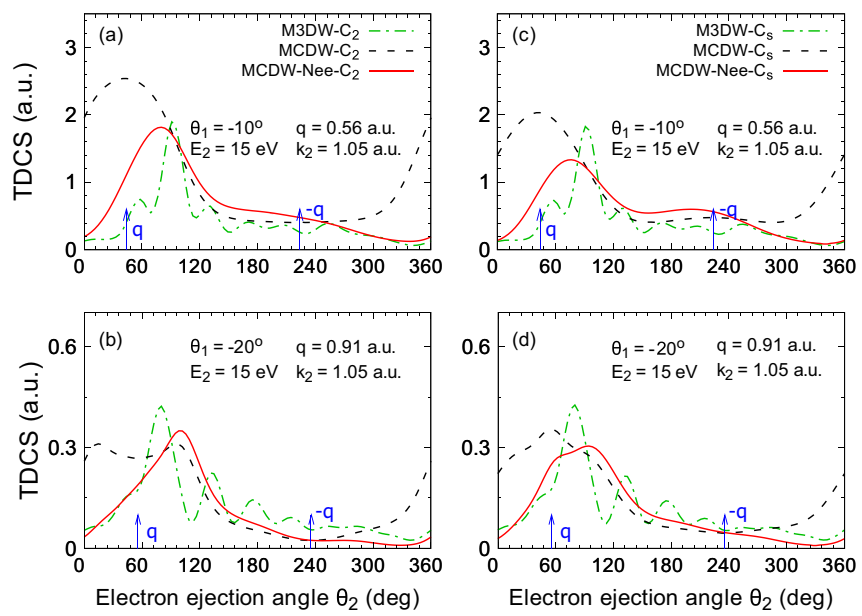

FIG. 2. MCDW (dashed lines), MCDW-Nee (solid lines), and M3DW (dashed-dotted lines) calculated TDCSs in the scattering plane for the HOMO of $C_{2}$ (left column) and $C_{s}$ (right column) conformers of THF under the kinematics of $\theta_{1}=-10^{\circ}$ and $E_{2}=15 \mathrm{eV}$ (top row), and $\theta_{1}=-20^{\circ}$ and $E_{2}=15 \mathrm{eV}$ (bottom row). Ionization energy IE and projectile energy $E_{0}$ are 9.8 and $91 \mathrm{eV}$, respectively. The vertical arrows indicate the momentum transfer direction $q$ and its opposite $-q$.

\section{3D TDCS and definition of cutting planes}

The summed theoretical TDCS of two conformers will be used in the following discussions to compare with the experimental data. Figure 3 presents the theoretical threedimensional (3D) surface plot of the TDCSs at a projectile scattering angle of $\theta_{1}=-10^{\circ}$ as a function of the emission direction of the slow ejected electron with $E_{2}=6 \mathrm{eV}$ calculated using the MCDW-Nee model. In this 3D plot, the TDCS for a particular direction is defined as the distance from the origin to the surface. The projectile $\left(\vec{k}_{0}\right)$ enters from the bottom and is scattered to the left with momentum vector $\vec{k}_{1}$ (hence the

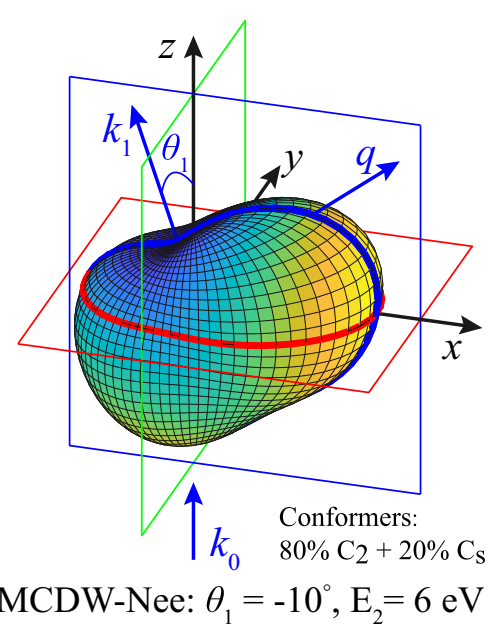

FIG. 3. Theoretical MCDW-Nee three-dimensional image of the TDCSs at a projectile scattering angle of $\theta_{1}=-10^{\circ}$ and ejectedelectron energy $E_{2}=6 \mathrm{eV}$. Ionization energy IE and projectile energy $E_{0}$ are 9.8 and $91 \mathrm{eV}$, respectively. The theoretical results are calculated including the two conformers of THF. 
minus in the notation for the scattering angle $\theta_{1}$ ). These two vectors define the scattering $x z$ plane as indicated by the blue solid frame in Fig. 3. The asymmetric kinematic condition is adopted where the fast outgoing electron can be safely considered as the scattered projectile and the momentum transferred to the target $\vec{q}$ indicated in Fig. 3 is defined as $\vec{q}=\vec{k}_{0}-\vec{k}_{1}$.

Two basic features are observed in the 3D TDCS. Electrons emitted roughly in the direction of the momentum transfer form the well-known binary lobe while electrons emitted in the opposite direction form the recoil lobe. The binary lobe can be explained as the binary collision between the projectile and target electron while the recoil lobe is due to backward rescattering of the ejected electron in the molecular potential. At the present low impact energy $(91 \mathrm{eV})$, the PCI effect is strong (as expected) and, consequently, the repulsive force between the two electrons rotates the binary peak to larger scattering angles relative to the momentum transfer direction. Compared to the 3D TDCS for water molecules at a similar impact energy [40,52], the present result depicts a modified binary peak shape as well as filling of the minimum separating the binary from the recoil lobe leading to a somehow isotropic distribution. This might result from the multicenter nature of the residual ion potential.

For a quantitative study of TDCS over a large range of the measured phase space we present cuts through the 3D TDCS for the three different planes of the ejected electron as indicated in Fig. 3. In addition to the scattering plane, the half-perpendicular plane and the full-perpendicular plane are defined as follows: The half-perpendicular $y z$ plane (green frame) contains the incoming beam axis and is perpendicular to the scattering plane. The full-perpendicular $x y$ plane (red frame) is perpendicular to the incoming projectile beam direction. The studied kinematic conditions correspond to scattering angles of $\theta_{1}=-10^{\circ},-15^{\circ}$, and $-20^{\circ}$ and ejected-electron energies $E_{2}=6$ and $15 \mathrm{eV}$, respectively. The experimental cross sections are not absolute: The different kinematics are measured in the same experimental run and thus are cross normalized. Thereby, a single common factor is sufficient for normalization. The experiment and MCDW, MCDW-Nee results are normalized to the binary lobe of the M3DW calculation at the scattering angle of $\theta_{1}=-10^{\circ}$ and ejected-electron energy of $E_{2}=15 \mathrm{eV}$ [see Fig. 4(b)]. This factor was subsequently applied to all other kinematics and planes.

\section{TDCSs for the three cutting planes}

In Figs. 4-6, the experimental cross sections are presented as a function of the ejected-electron emission angles. The scattering plane (Fig. 4) cuts through the binary and the recoil lobes and it contains the momentum transfer vector indicated in the diagrams by arrows. In this plane, the well-known binary and recoil patterns can be better observed at a higher ejected energy of $E_{2}=15 \mathrm{eV}$ (right column), in particular for the scattering angle of $\theta_{1}=-10^{\circ}$ [Fig. 4(b)], while for $E_{2}=$ $6 \mathrm{eV}$ (left column) these two patterns tend to overlap, making it difficult to distinguish between them.

In the half-perpendicular plane (Fig. 5), there is an indication of a three-lobe structure, i.e., a central maximum at $\theta_{2}=180^{\circ}$ and two maxima at $\theta_{2} \sim 90^{\circ}$ and $270^{\circ}$, which can

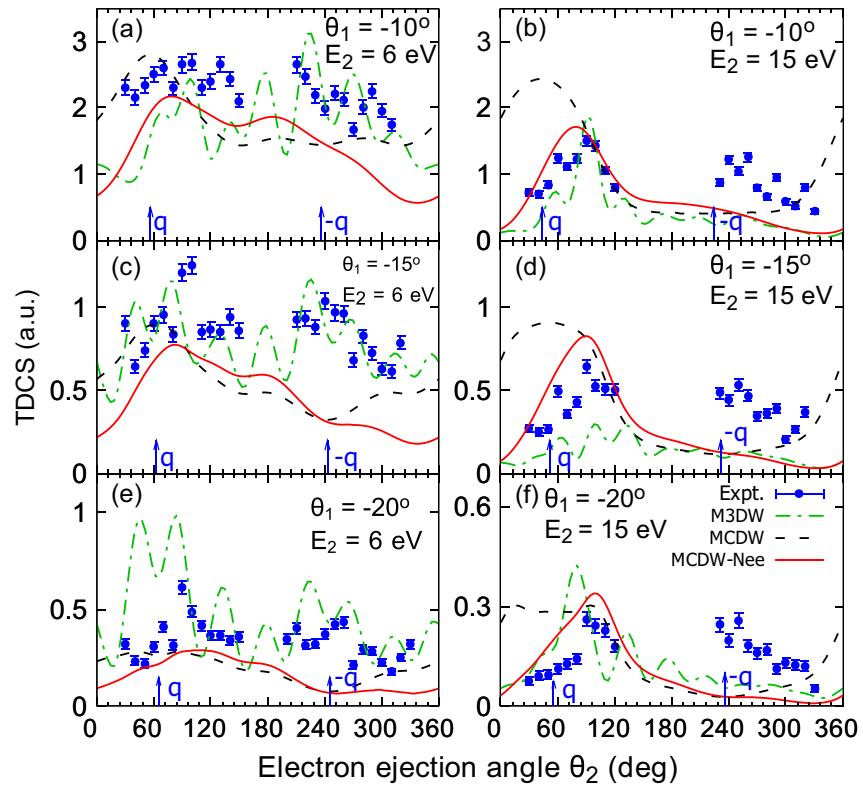

FIG. 4. Comparison between experimental TDCS (solid circles with error bars), with MCDW (dashed lines), MCDW-Nee (solid lines), and $\mathrm{M} 3 \mathrm{DW}$ (dashed-dotted lines) calculations for electronimpact $\left(E_{0}=91 \mathrm{eV}\right)$ ionization of the HOMO of THF presented as a function of the ejected-electron $\left(e_{2}\right)$ emission angle at scattering angles $\theta_{1}=-10^{\circ}$ (top row), $-15^{\circ}$ (middle row), and $-20^{\circ}$ (bottom row) for ejected-electron energies $E_{2}=6 \mathrm{eV}$ (left column), and $15 \mathrm{eV}$ (right column), and ionization energy $\mathrm{IE}=9.8 \mathrm{eV}$. The vertical arrows indicate the momentum transfer direction $q$ and its opposite $-q$. The results are for the scattering plane, i.e., the $x z$ plane of Fig. 3.

be more clearly seen for the ejected-electron energy of $6 \mathrm{eV}$ in the left columns of Fig. 5. It can be seen in the 3D plot of Fig. 3 that this plane cuts through the binary lobe which results in two symmetric maxima at $\theta_{2} \sim 90^{\circ}$ and $270^{\circ}$, respectively, which may result from high-order projectile-target interactions [53]. In addition, the recoil lobe gives rise to the central maximum at $\theta_{2}=180^{\circ}$. In this plane PCI acts strongest for emission angles near $0^{\circ}$ and $360^{\circ}$.

In the full-perpendicular plane (Fig. 6), the ejected electron's polar angle is fixed to $\theta_{2}=90^{\circ}$ and the azimuthal angle $\phi_{2}$ is varied. In this plane, the experimental angular acceptance covers the entire angle range, i.e., $\phi_{2}$ from $0^{\circ}$ to $360^{\circ}$, and the cross sections are again symmetric with respect to $180^{\circ}$. The experimental TDCSs show less structures compared to the other two planes. Here, a maximum intensity is observed at $\phi_{2} \sim 0^{\circ}$, particularly for the lower ejected energies (left column), which corresponds to the binary lobe, while the recoil lobe at $\phi_{2} \sim 180^{\circ}$ shows a minimum or a flat distribution. In this plane the influence of PCI appears to be small over the entire angular range.

The experimental TDCSs in the scattering plane, i.e., the $x z$ plane of Fig. 3, are compared with the theoretical MCDW, MCDW-Nee, and M3DW results in Fig. 4. For all kinematics, the MCDW and MCDW-Nee calculations exhibit double-lobe structures, the binary lobe near $\mathbf{q}$ and the recoil lobe near $-\mathbf{q}$ directions which generally reproduce the experiment. The MCDW calculation provides too high of an intensity at the 


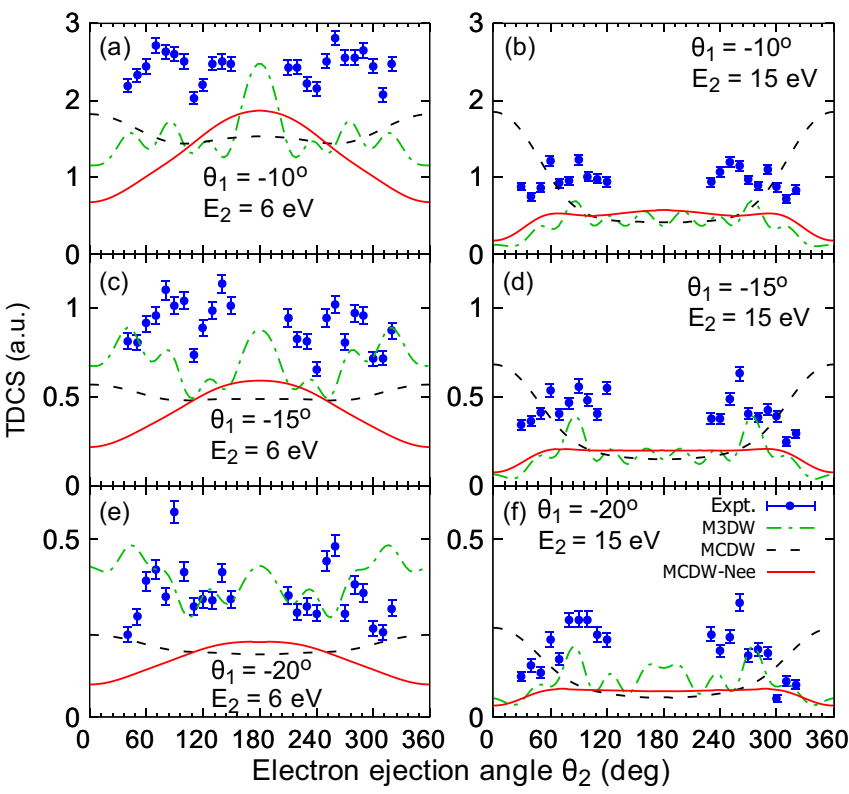

FIG. 5. Same as Fig. 4 but for the half-perpendicular plane, i.e., the $y z$ plane of Fig. 3 .

electron ejection angle $\theta_{2}$ near $0^{\circ}$ which is in the vicinity of the scattered projectile direction. The MCDW-Nee gives an improved description of the experimental TDCS by inclusion of the PCI effect. On one hand, the intensities of the TDCS at $\theta_{2}=0^{\circ}$ and $360^{\circ}$ are reduced. On the other hand, due to the repulsive force between the two outgoing electrons, the binary peak is shifted to larger angles for all kinematic conditions. The MCDW-Nee shows better agreement with experiment than MCDW, indicating that the PCI effect is very important for low-energy experiments.

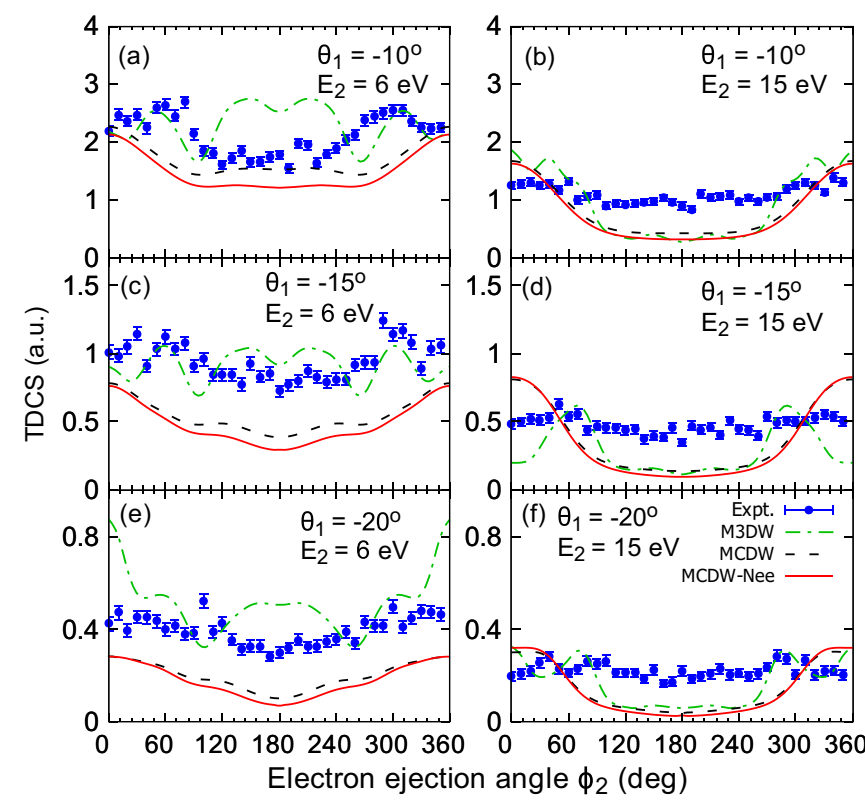

FIG. 6. Same as Fig. 4 but for the full-perpendicular plane, i.e., the $x y$ plane of Fig. 3 .
For the M3DW results, in addition to the binary and recoil lobes, there are additional structures which partly fit the experimental data at a low ejected energy of $6 \mathrm{eV}$. For an incident projectile energy of $250 \mathrm{eV}$ [14,15,18], the M3DW provided a fairly good agreement with experiment concerning the relative intensity between binary and recoil lobes, except for a few cases. Overall, both M3DW and MCDW-Nee agree qualitatively with the experimental data in the scattering plane.

Figure 5 shows the comparison of TDCS between experiment and theoretical calculations in a half-perpendicular plane, i.e., the $y z$ plane in Fig. 3. In this case, a symmetric TDCS is required about $\theta_{2}=180^{\circ}$, which is indeed seen in both theory and experiment. Concerning the angular dependence of cross sections at $\theta_{2}$ near $0^{\circ}$, the MCDW-Nee is in much better agreement with experiment than the MCDW, indicating the strong PCI effect near the projectile forward direction. Here, the MCDW predicts a flat distribution at $\theta_{2}$ near $180^{\circ}$ and a maximum at $\theta_{2}$ near $0^{\circ}$. In all panels, the observed two maxima at $\theta_{2} \sim 90^{\circ}$ and $270^{\circ}$ are not reproduced by either the MCDW or the MCDW-Nee calculations. The M3DW theory is able to reproduce the observed three-lobe structure, while deviations in the magnitude of the three-lobe structure are visible for the case of $\theta_{1}=-10^{\circ}$ and $-15^{\circ}$ in the top and middle rows of Fig. 5.

Figure 6 shows the TDCS in the full-perpendicular plane, i.e., the $x y$ plane. The MCDW and MCDW-Nee models are in fairly good agreement with the experiment. The almost identical results of the MCDW and MCDW-Nee indicate that the PCI effect is rather small in this plane which was also observed in the results of $\mathrm{CO}_{2}$ at the impact energy of $100 \mathrm{eV}$ [54]. As for M3DW calculations, the predicted TDCS intensities are in general agreement with experiment. The M3DW predicts a structure which is generally not seen in the experimental data although there is some indication of the structure in the $-15^{\circ}$ data for $6 \mathrm{eV}$. Concerning the overall TDCS distributions, the MCDW and MCDW-Nee models are better than M3DW in this plane.

\section{CONCLUSION}

We reported a comprehensive study of the electron-impact ionization dynamics of the HOMO of THF molecules at a low projectile energy of $91 \mathrm{eV}$. The relative abundance of $80 \%$ : $20 \%$ for the $C_{2}$ to $C_{s}$ conformers for THF was determined based on the supersonic gas jet condition in the experiment. The measured three-dimensional triple-differential cross sections (TDCSs) obtained experimentally were internormalized across all scattering angles of $\theta_{1}$ from $-10^{\circ}$ to $-20^{\circ}$ and ejected-electron energies of $E_{2}$ from 6.0 to $15.0 \mathrm{eV}$, thus providing a stringent test for the theoretical models. The experimental data were compared with predictions from the MCDW, MCDW-Nee, and M3DW models.

The MCDW model provides reasonable agreement with experiment only for the TDCS in the full-perpendicular plane, where the PCI effects do not play a significant role. The predictions from the MCDW-Nee and M3DW models, where PCI is accounted for via the Ward-Macek approximation for MCDW-Nee and using an exact treatment of PCI for M3DW, improve the results in the scattering and half-perpendicular 
planes. This clearly indicates that PCI effects play a very important role in the present low-energy ionization of molecules. However, there is a systematic discrepancy between MCDWNee and experiment in the half-perpendicular plane where the observed two peaks at $\theta_{2} \sim 90^{\circ}$ and $270^{\circ}$ are not predicted by the MCDW-Nee model while they are predicted by the M3DW model. The M3DW predicts significant oscillations in the TDCS which agree partly with the measurements in the scattering plane and for the $E_{2}=6 \mathrm{eV}$ kinematics. These oscillation structures may result from interference of the integrated complex waves. It is also to be noted that in the MCDW method the transition matrix is calculated in the molecular frame and the TDCS is obtained by taking an analytical average over all molecular orientations. In the M3DW approach, the molecular orientation averaging is accounted for in the transition matrix where the orientation-averaged initial bound-state Dyson orbital is adopted.

Finally, we point out that the two calculations using MCDW-Nee and M3DW models differ from each other in both the relative shape and the magnitude of the cross sections. This illustrates the fact that the theoretical treatment of the electron-impact ionization of molecules is very complicated and the results are very sensitive to the details of the model employed. Both MCDW-Nee and M3DW calculations are far from satisfactory for reproducing the triple-differential cross sections of the THF molecule at low projectile energy. More sophisticated models which can calculate more accurate distorted waves or replace plane waves with distorted waves $[40,52,55]$ are required to describe the ionization dynamics of complex molecules.

\section{ACKNOWLEDGMENTS}

This work is jointly supported by the National Natural Science Foundation of China (Grants No. 11774281, No. 11534011, No. 11974272, and No. 11327404) and the National Key Research and Development Program of China (Grant No. 2017YFA0402302), and the DFG Project No. RE 2966/3-1. E.W. acknowledges support from a fellowship from the Alexander von Humboldt Foundation.
[1] E. Alizadeh, T. M. Orlando, and L. Sanche, Annu. Rev. Phys. Chem. 66, 379 (2015).

[2] K. Bartschat and M. J. Kushner, Proc. Natl. Acad. Sci. USA 113, 7026 (2016).

[3] L. Campbell and M. Brunger, Int. Rev. Phys. Chem. 35, 297 (2016).

[4] B. Boudaïffa, P. Cloutier, D. Hunting, M. A. Huels, and L. Sanche, Science 287, 1658 (2000).

[5] M. A. Huels, B. Boudaiffa, P. Cloutier, D. Hunting, and L. Sanche, J. Am. Chem. Soc. 125, 4467 (2003).

[6] S. M. Pimblott and J. A. LaVerne, Radiat. Phys. Chem. 76, 1244 (2007).

[7] E. Wang, X. Ren, W. Baek, H. Rabus, T. Pfeifer, and A. Dorn, Nat. Commun. 11, 2194 (2020).

[8] I. I. Fabrikant, S. Eden, N. J. Mason, and J. Fedor, Adv. At., Mol., Opt. Phys. 66, 545 (2017).

[9] J. Kočišek, A. Pysanenko, M. Fárník, and J. Fedor, J. Phys. Chem. Lett. 7, 3401 (2016).

[10] P. Papp, P. Shchukin, J. Kočíšek, and S. Matejčík, J. Chem. Phys. 137, 105101 (2012).

[11] H. Ehrhardt, K. Jung, G. Knoth, and P. Schlemmer, Z. Phys. D: At., Mol. Clusters 1, 3 (1986).

[12] T. N. Rescigno, M. Baertschy, W. A. Isaacs, and C. W. McCurdy, Science 286, 2474 (1999).

[13] E. Ali, H. S. Chakraborty, and D. H. Madison, J. Chem. Phys. 152, 124303 (2020).

[14] C. J. Colyer, S. M. Bellm, B. Lohmann, G. F. Hanne, O. AlHagan, D. H. Madison, and C. G. Ning, J. Chem. Phys. 133, 124302 (2010).

[15] D. Jones, J. Builth-Williams, S. Bellm, L. Chiari, H. Chaluvadi, D. Madison, C. Ning, B. Lohmann, O. Ingólfsson, and M. Brunger, Chem. Phys. Lett. 572, 32 (2013).

[16] J. D. Builth-Williams, S. M. Bellm, L. Chiari, P. A. Thorn, D. B. Jones, H. Chaluvadi, D. H. Madison, C. G. Ning, B. Lohmann, G. B. da Silva, and M. J. Brunger, J. Chem. Phys. 139, 034306 (2013).
[17] E. Ali, X. G. Ren, A. Dorn, C. Ning, J. Colgan, and D. Madison, Phys. Rev. A 93, 062705 (2016).

[18] X. Xu, M. Gong, X. Li, S. B. Zhang, and X. Chen, J. Chem. Phys. 148, 244104 (2018).

[19] S. Tonzani and C. H. Greene, J. Chem. Phys. 125, 094504 (2006).

[20] B. C. Ibănescu, O. May, and M. Allan, Phys. Chem. Chem. Phys. 10, 1507 (2008).

[21] M. Dampc, E. Szymańska, B. Mielewska, and M. Zubek, J. Phys. B 44, 055206 (2011).

[22] X. Ren, T. Pflüger, M. Weyland, W. Y. Baek, H. Rabus, J. Ullrich, and A. Dorn, J. Chem. Phys. 141, 134314 (2014).

[23] M. Neustetter, M. Mahmoodi-Darian, and S. Denifl, J. Am. Soc. Mass Spectrom. 28, 866 (2017).

[24] X. Ren, E. Wang, A. D. Skitnevskaya, A. B. Trofimov, K. Gokhberg, and A. Dorn, Nat. Phys. 14, 1062 (2018).

[25] A. Milosavljevic, A. Giuliani, D. Sevic, M.-J. Hubin-Franskin, and B. Marinkovic, Eur. Phys. J. D 35, 411 (2005).

[26] C. Winstead and V. McKoy, J. Chem. Phys. 125, 074302 (2006).

[27] C. S. Trevisan, A. E. Orel, and T. N. Rescigno, J. Phys. B: At., Mol. Opt. Phys. 39, L255 (2006).

[28] M. Allan, J. Phys. B: At., Mol. Opt. Phys. 40, 3531 (2007).

[29] A. Gauf, L. R. Hargreaves, A. Jo, J. Tanner, M. A. Khakoo, T. Walls, C. Winstead, and V. McKoy, Phys. Rev. A 85, 052717 (2012).

[30] J. Gao, D. H. Madison, J. L. Peacher, A. J. Murray, and M. J. Hussey, J. Chem. Phys. 124, 194306 (2006).

[31] E. Ali, K. Nixon, A. Murray, C. Ning, J. Colgan, and D. Madison, Phys. Rev. A 92, 042711 (2015).

[32] M. Dürr, C. Dimopoulou, B. Najjari, A. Dorn, and J. Ullrich, Phys. Rev. Lett. 96, 243202 (2006).

[33] X. Ren, T. Pflüger, J. Ullrich, O. Zatsarinny, K. Bartschat, D. H. Madison, and A. Dorn, Phys. Rev. A 85, 032702 (2012).

[34] T. Pflüger, A. Senftleben, X. Ren, A. Dorn, and J. Ullrich, Phys. Rev. Lett. 107, 223201 (2011). 
[35] X. Ren, A. Senftleben, T. Pflüger, A. Dorn, K. Bartschat, and J. Ullrich, Phys. Rev. A 83, 052714 (2011).

[36] X. Ren, T. Pflüger, S. Xu, J. Colgan, M. S. Pindzola, A. Senftleben, J. Ullrich, and A. Dorn, Phys. Rev. Lett. 109, 123202 (2012).

[37] T. Pflüger, O. Zatsarinny, K. Bartschat, A. Senftleben, X. Ren, J. Ullrich, and A. Dorn, Phys. Rev. Lett. 110, 153202 (2013).

[38] X. Ren, S. Amami, O. Zatsarinny, T. Pflüger, M. Weyland, W. Y. Baek, H. Rabus, K. Bartschat, D. Madison, and A. Dorn, Phys. Rev. A 91, 032707 (2015).

[39] X. Ren, S. Amami, O. Zatsarinny, T. Pflüger, M. Weyland, A. Dorn, D. Madison, and K. Bartschat, Phys. Rev. A 93, 062704 (2016).

[40] X. Ren, S. Amami, K. Hossen, E. Ali, C. G. Ning, J. Colgan, D. Madison, and A. Dorn, Phys. Rev. A 95, 022701 (2017).

[41] S. Zhang, X. Y. Li, J. G. Wang, Y. Z. Qu, and X. Chen, Phys. Rev. A 89, 052711 (2014).

[42] X. Li, M. Gong, L. Liu, Y. Wu, J. Wang, Y. Qu, and X. Chen, Phys. Rev. A 95, 012703 (2017).

[43] X. Li, X. Ren, K. Hossen, E. Wang, X. Chen, and A. Dorn, Phys. Rev. A 97, 022706 (2018).

[44] X. Ren, E. Jabbour Al Maalouf, A. Dorn, and S. Denifl, Nat. Commun. 7, 11093 (2016).
[45] J. Ullrich, R. Moshammer, A. Dorn, R. Dörner, L. P. H. Schmidt, and H. Schmidt-Böcking, Rep. Prog. Phys. 66, 1463 (2003).

[46] D. H. Madison and O. Al-Hagan, J. At. Mol. Opt. Phys. 2010, 367180 (2010).

[47] S. J. Ward and J. H. Macek, Phys. Rev. A 49, 1049 (1994).

[48] C. G. Ning, Y. R. Huang, S. F. Zhang, J. K. Deng, K. Liu, Z. H. Luo, and F. Wang, J. Phys. Chem. A 112, 11078 (2008).

[49] S. M. Park, Y. R. Lee, D. W. Kang, H. L. Kim, and C. H. Kwon, Phys. Chem. Chem. Phys. 19, 30362 (2017).

[50] M. J. Frisch, G. W. Trucks, H. B. Schlegel, G. E. Scuseria, M. A. Robb, J. R. Cheeseman, G. Scalmani, V. Barone, G. A. Petersson, H. Nakatsuji, X. Li, M. Caricato, A. V. Marenich, J. Bloino, B. G. Janesko, R. Gomperts, B. Mennucci, H. P. Hratchian, J. V. Ortiz, A. F. Izmaylov et al., Gaussian 16 Revision A.03 (Gaussian Inc., Wallingford, CT, 2016).

[51] M. A. Coplan, J. H. Moore, and J. P. Doering, Rev. Mod. Phys. 66, 985 (1994).

[52] M. Gong, X. Li, S. B. Zhang, S. Niu, X. Ren, E. Wang, A. Dorn, and X. Chen, Phys. Rev. A 98, 042710 (2018).

[53] X. Ren, A. Senftleben, T. Pflüger, A. Dorn, K. Bartschat, and J. Ullrich, J. Phys. B: At., Mol. Opt. Phys. 43, 035202 (2010).

[54] K. Hossen, X. Ren, E. Wang, M. Gong, X. Li, S. B. Zhang, X. Chen, and A. Dorn, J. Phys. B 51, 215201 (2018).

[55] E. Acebal and S. Otranto, Phys. Rev. A 98, 012703 (2018). 